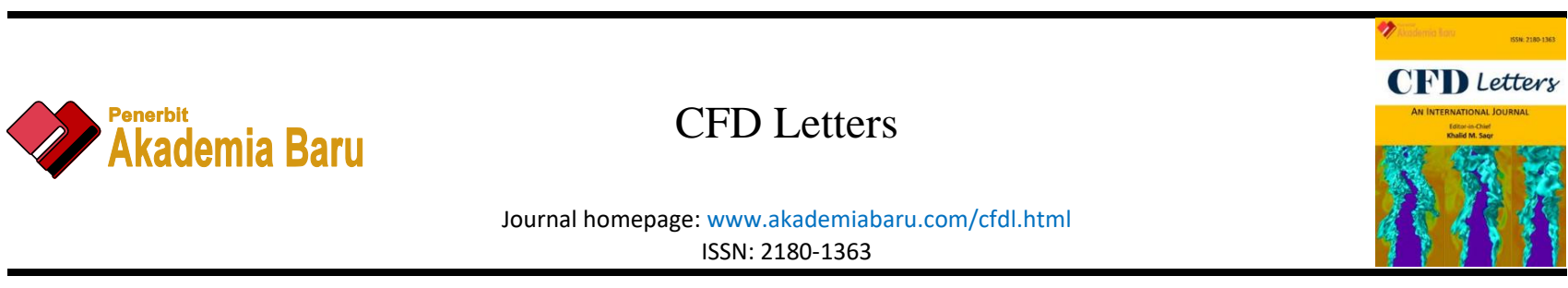

\title{
The Effect of Grid Skewness on Non-Unified Compact Residual Distribution Methods for Scalar Advection Diffusion Problems
}

\author{
Hazim Fadli Aminnuddin ${ }^{1,}{ }^{*}$, Farzad Ismail ${ }^{2}$, Akmal Nizam Mohamed ${ }^{1}$, Kamil Abdullah $^{1}$ \\ Faculty of Mechanical and Manufacturing, Universiti Tun Hussein Onn Malaysia, Parit Raja, 86400, Johor, Malaysia \\ School of Aerospace Engineering, Engineering Campus, Universiti Sains Malaysia, Nibong Tebal, 14300, Pulau Pinang, Malaysia
}

\section{ARTICLE INFO}

\section{Article history:}

Received 23 January 2020

Received in revised form 18 March 2020

Accepted 23 March 2020

Available online 29 March 2020

Keywords:

Residual distribution; flux-difference; advection-diffusion; non-unified; grid skewness

\section{ABSTRACT}

\begin{abstract}
In this paper, the newly developed residual distribution (RD) method called the FluxDifference approach is combined with the Galerkin method to solve the advectiondiffusion equation in separate (non-unified) manner. It is due to the incapability of variation grid skewness in finite volume. This Flux-Difference RD method maintains a compact stencil and the whole process of solving advection-diffusion do not require additional equations. In order to improve the order of accuracy losses by the classic RD schemes, the present scheme will be tested using non-unified manners. The numerical results show that the Flux-Difference RD method preserves second-order accuracy up to about skewness 0.4 but drops to about 1.5 orders accurate when grid skewness is 0.6 .
\end{abstract}

Copyright $\odot 2020$ PENERBIT AKADEMIA BARU - All rights reserved

\section{Introduction}

The finite volume (FV) method is one of the commonly used discretization methods in the modern Computational Fluid Dynamics (CFD) simulations [1-2]. However, FV have difficulties to capture the multi-dimensional physics of wave propagation for hyperbolic equation and suffer from severe results degradation on skewed grids as reported in the past researches [3-5]. Thus, RD methods were introduced as an alternative to the FV methods by Roe [6]. Discretization on RD method is based on cell vertex solutions and cell residuals. RD methods are known to be less sensitive to grid variations and have been proven by Chizari, Ismail and Abgrall [7-9] for advection problems. RD methods are also more compact which allows a more efficient parallel computation [10] and have a natural platform to incorporate multidimensional fluid physics stated by Deconinck et al., [11]. However, there is still lot to be done for RD methods. Most RD methods are developed from mainly steadystate inviscid equations. Most of these methods will be at best first order accurate in space for

\footnotetext{
* Corresponding author.

E-mail address: hazimfadli91@gmail.com (Hazim Fadli Aminnuddin)
} 
unsteady calculations even if they were high order accurate for steady-state problems unless there is a costly implicit sub-iterative process being applied at every time-step [12].

In order to reach the full potential of RD method, Ismail and Chizari [13] have developed a new class of RD method called Flux-Difference approach which ensures automatic conservation of the primary variables without any dependence on cell-averaging for any well-posed equations and preserves the spatial second-order accuracy on unsteady problems using any consistent explicit time integration scheme. However, since these flux-difference RD methods are new, very little has been done to understand the inherent properties of the scheme. As a matter of interest, the present study will be focusing on advection-diffusion problems. Owing to the different physics of advection and diffusion, it is perhaps best to discretize them in a non-unified (separate) manner. However, the RD and Galerkin approach for non-unified manner still not fully resolved. These issue have been encountered by the past research [14-16] where the results show that an accuracy loss even on ideal grids. To overcome this issue, Singh et al., [17] have used the Flux-Difference RD method as an alternative to the classic RD method. The results are very promising where the order of accuracy is preserved at second order accurate. But the grid skewness used is only 0.0 and there are no information on the highly skewed grid.

Thus, in this paper, the Flux-Difference approach will be tested herein to see if second order accurate is preserved not only for ideal grids but also for highly skewed grids. The objectives of this paper is to provide the information on highly skewed grid and the result comparison between grids while maintaining all variables. The paper is organized as the following. Section 2 presents the classic scalar RD discretization for advection and diffusion terms while Sect. 3 presents the Flux-Difference RD method for advection-diffusion. The numerical results will be demonstrated in Sect. 4 while Sect. 5 concludes this research paper.

\section{Methodology}

\subsection{Residual-Distribution Approach for Advection-Diffusion}

$\mathrm{RD}$ schemes are numerical methods that involve two steps. The first is the residual calculation, followed by the distribution of the residuals to nodes where the residual drives the changes of the solution. Consider, as an example, the two dimensional scalar advection- diffusion equations.

$u_{t}+\nabla \cdot F=\nabla \cdot G$

where,

$F=u \lambda=(a u) \hat{\imath}+(b u) \hat{\jmath}$

$G=\left(v u_{x}\right) \hat{\imath}+\left(v u_{y}\right) \hat{\jmath}$

The $\lambda$ in the equation is the characteristic vector such that $\lambda=a \hat{\imath}+b \hat{\jmath}$ where $a$ and $b$ are the advection speeds in $x$ and $y$ direction and $v$ is the positive diffusion coefficient. Then the computational domain is divided into a sets of triangle $\{T\}$ and the solution values at nodes will be stored by the set of nodes $\{J\}$. Figure 1 shows the details of the normal direction, $\boldsymbol{n}$ and also the median dual-area, $S_{i}$. 

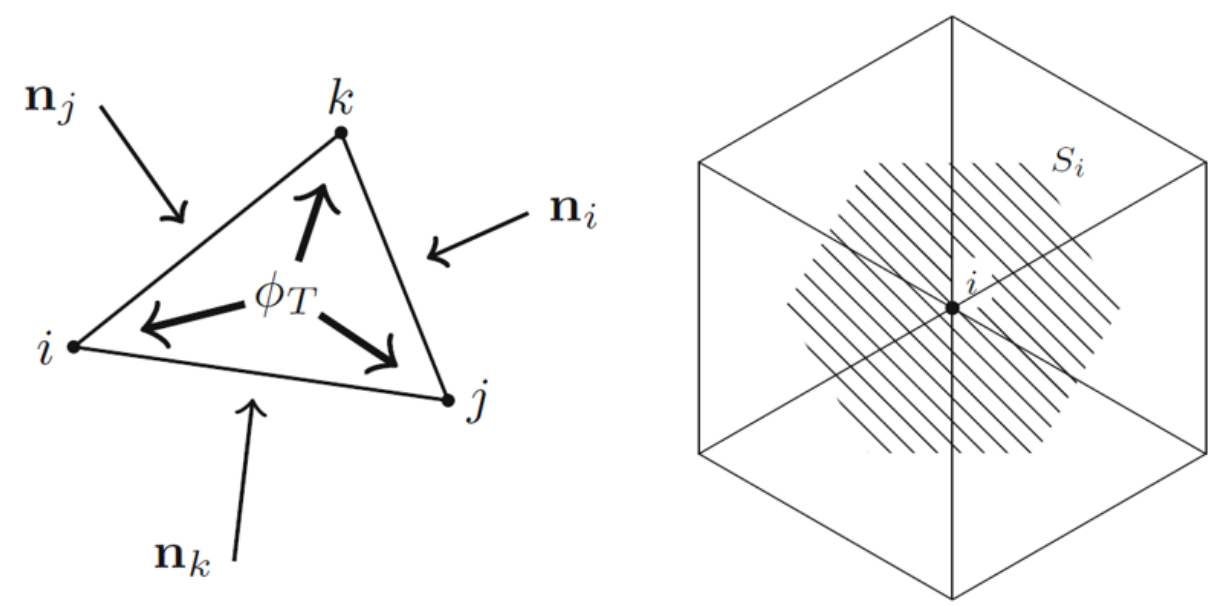

Fig. 1. Residual distributed over triangular mesh

The main concept of the RD method is finding the sub-residuals (or signals) for each point from the total residual of a cell (element) inward scaled normal. In order to mimic the correct physics of the problem, the present study discretized the inviscid terms and viscous terms separately. This will allow a multidimensional upwind solution update from inviscid residual, $\emptyset_{i n v}^{T}$ and an isotropic distribution of viscous residual, $\emptyset_{v i s}^{T}$ to update the solution nodes. As for the advection discretization, by uniting the contributions from all the triangles surrounding node $i$, the conservative update can be written as in Eq. (4) where the scalar distribution coefficients, $\beta_{i}^{T}$ sums up to unity to achieve consistency.

$S_{i} \frac{d u_{i}}{d t}=\sum_{T, i \in T} \emptyset_{i, i n v}^{T}=\sum_{T, i \in T} \beta_{i}^{T} \emptyset_{i n v}^{T}$

Meanwhile, for the viscous discretization, the Finite Element (FE) Galerkin approach has been implemented. Thus, $\emptyset_{v i s}^{T}$ can be represented as Eq. (5) where $v$ is a cell averaged and $A_{T}$ is the area of the triangular element.

$\emptyset_{i, v i s}^{T}=-\frac{v}{4 A_{T}} \sum_{j=1}^{3} u_{j} n_{j} . n_{i}$

By treating the advection and diffusion residual separately, the conserved, semi-discrete form of the governing equation for node $i$ is of the form below.

$S_{i} \frac{d u_{i}}{d t}-\sum_{T, i \in T}\left(\varnothing_{i, i n v}^{T}+\emptyset_{i, v i s}^{T}\right)=0$

\subsection{Flux-Difference Approach for Advection-Diffusion}

The Flux-Difference approach which is the main interest for the present study evaluates the residual for a triangular element based on nodal flux values. This approach consists of two components: isotropic signals and artificial signals as shown in Figure 2. The new RD signal distribution scheme for node $i$ of a triangular element $T$ can be written as $\emptyset_{i}=\emptyset_{i}^{i s o}+\emptyset_{i}^{\text {art }}$. The isotropic signal, $\emptyset_{i}^{i s o}$ is obtained by the trapezoidal integration of the total residual within an element distributed equally to each node. $\emptyset_{i}^{a r t}$ is the artificial signals. $\alpha, \beta$ and $\gamma$ are the free parameter and $\beta$ is set to zero for simplicity [6]. With these choices, the Flux-Difference RD approach is a centraltype method with artificial terms to stabilize it. Observe that $(\alpha, \gamma)$ are coefficients which are 
dependent on a length-scale factor of which can be used to achieve high-order accuracy as shown in Ref. [12]. By Using $(\alpha, \gamma)=O\left(h^{q}\right)$ with $q=1$ will yield another second order method defined as FluxDifference approach [12].

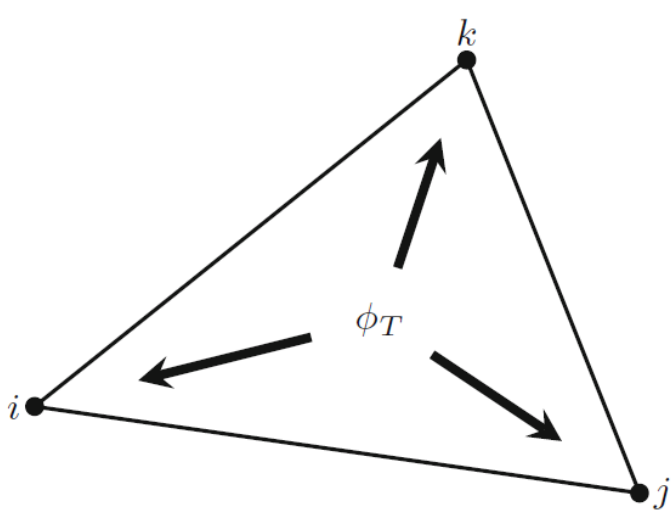

(a)

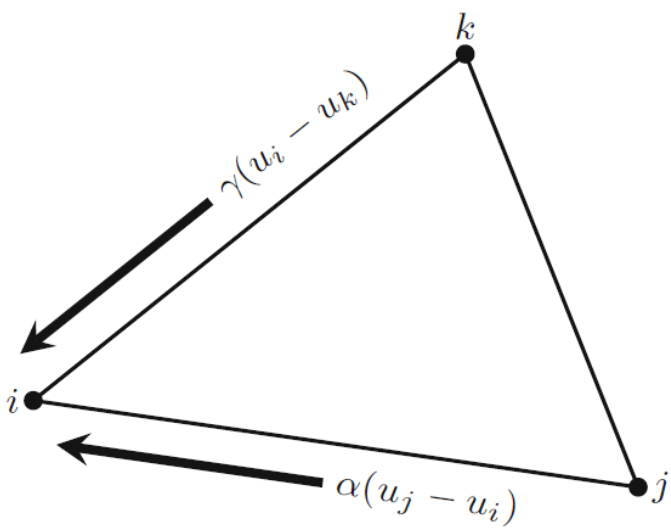

(b)

Fig. 2. Isotropic and artificial signal distribution to node i. (a) Isotropic signals and (b) Artificial signals

\subsection{Computational Domain and Boundary Condition}

The Flux Different RD approach and classic RD approach (Low Diffusion Advection-LDA) were tested on a steady two-dimensional linear advection-diffusion case. The grid used was equilateral grid using 101, 201 and 301 grid points (8989, 35175 and 78862 number of nodes) with variation of grid skewness ranged from 0.0 to 0.8 as shown in Figure 3 . Meanwhile, the boundary condition was set to the exact solution for all sides of a square domain $[0,1] \times[0,1]$ and was employed with a CFL $=0.1$ [15]. As for the benchmarking, Figure 4 shows the comparison of the exact solution between the present study and the work of Nishikawa [15]. Based on the figure, the present study has similar plot as in the previous work.

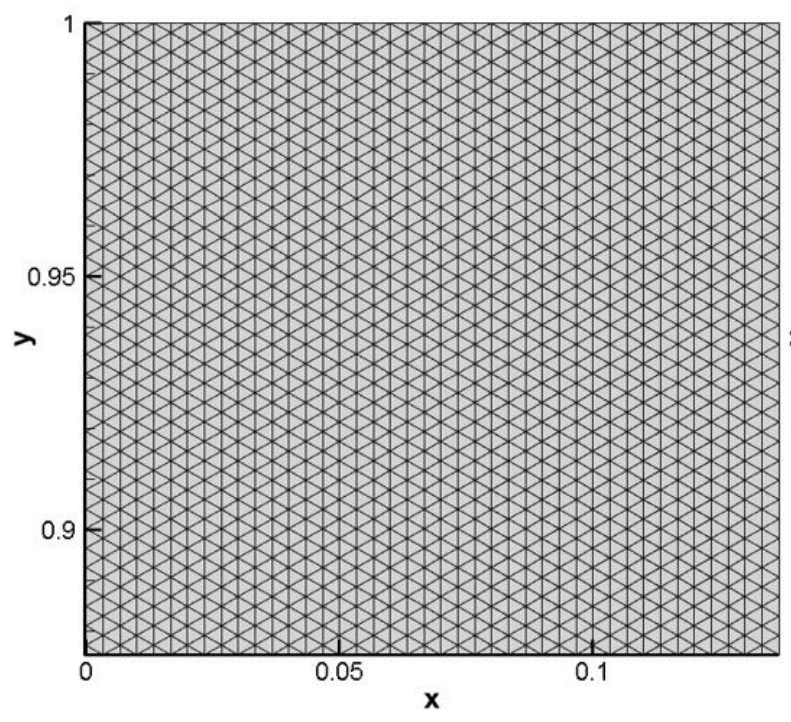

(a)

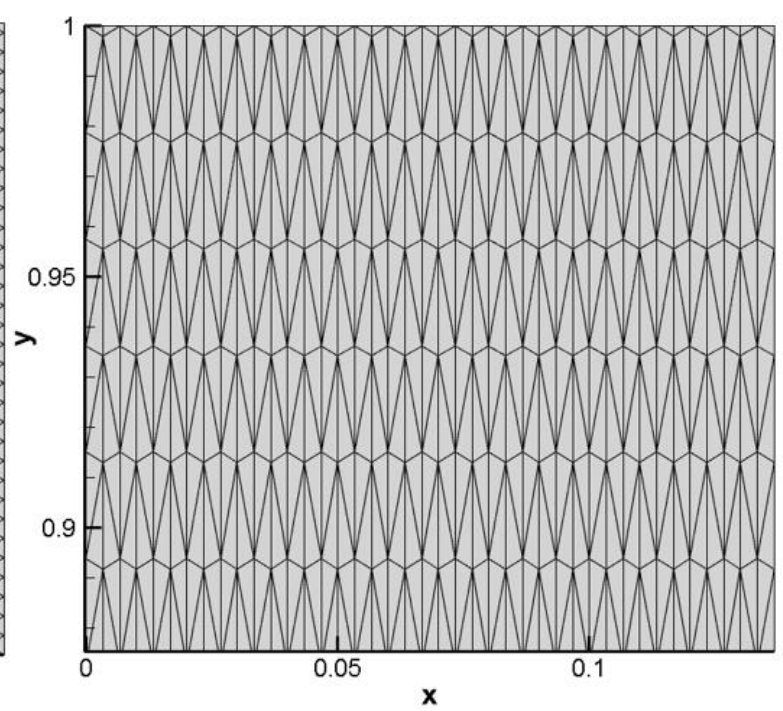

(b)

Fig. 3. Comparison of grid skewness; (a) Grid skewness 0.0 and (b) Grid skewness 0.8 


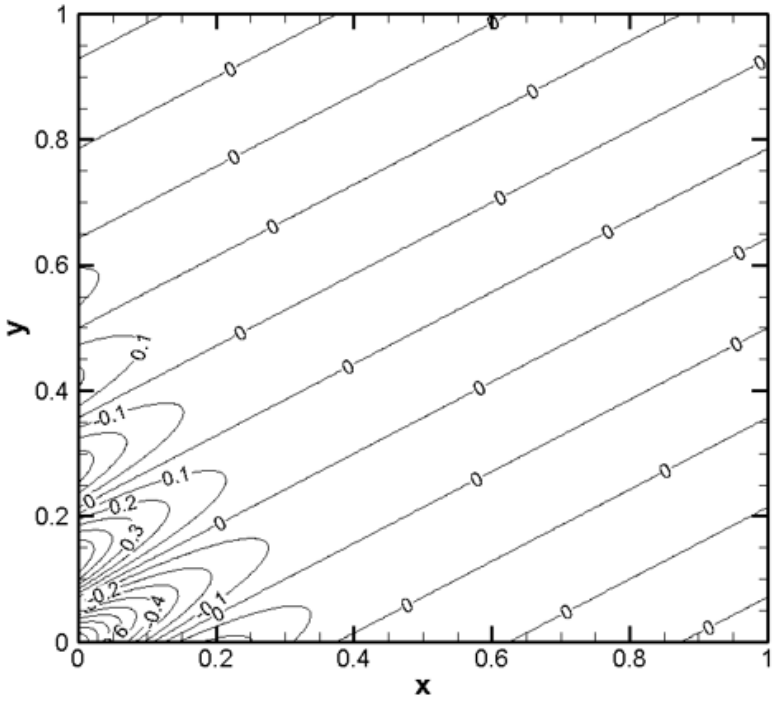

(a)

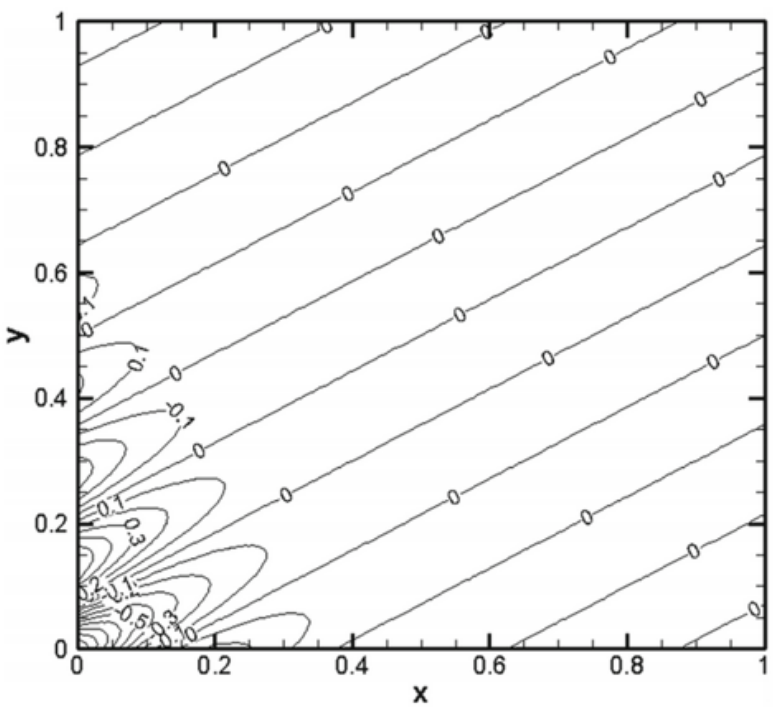

(b)

Fig. 4. The comparison of the exact solution from (a) present study and (b) past work of Nishikawa [15]

\section{Results}

Figure 5. demonstrates the error contour for the classic RD method (LDA). The error plotted indicates the difference between the exact solutions with the numerical solution. Based on the figure, the pattern is non-uniform for the coarse grid 101 . On the grid skewness 0.0 till 0.4 , the error contour distribution is observed to be smaller and narrower. But, this error distribution becomes larger as it reachs 0.6 grid skewness. However as the grid goes finer (grid 201 and 301), the pattern is uniform where the error distribution region mitigates when the skewness increasing. As for grid comparison, all considered cases produce same pattern where all the finer grids tend to produce less error for all value of grid skewness.
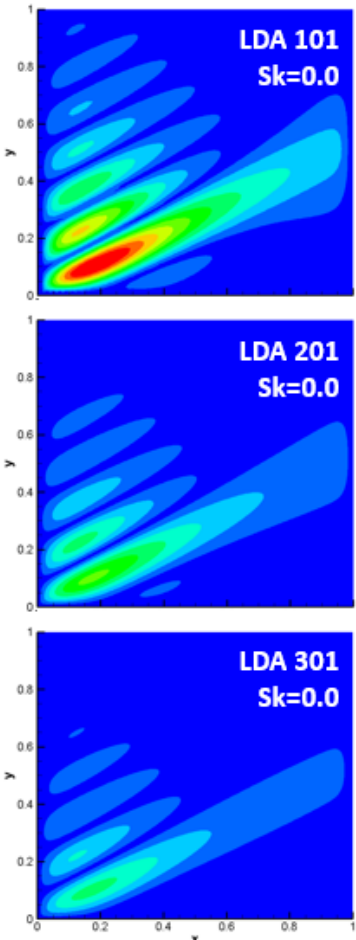
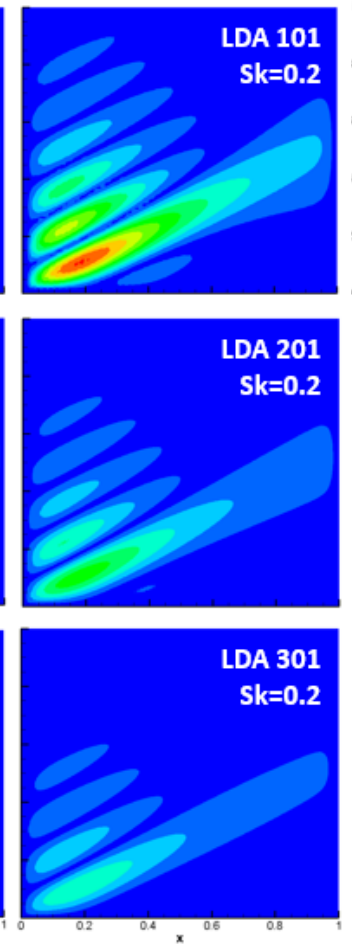

Fig. 5. Error contour for Classic LDA cases
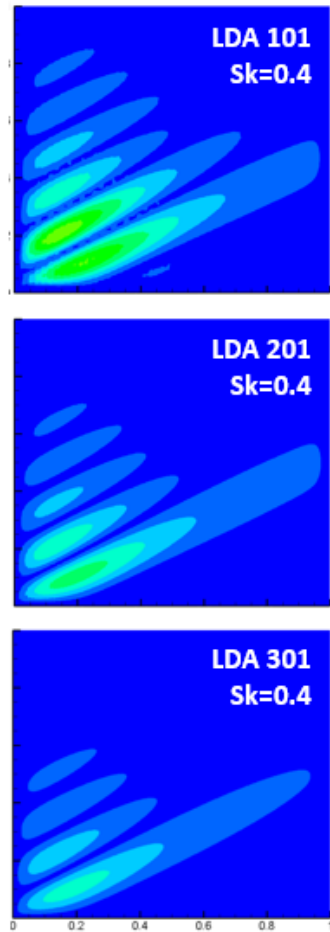
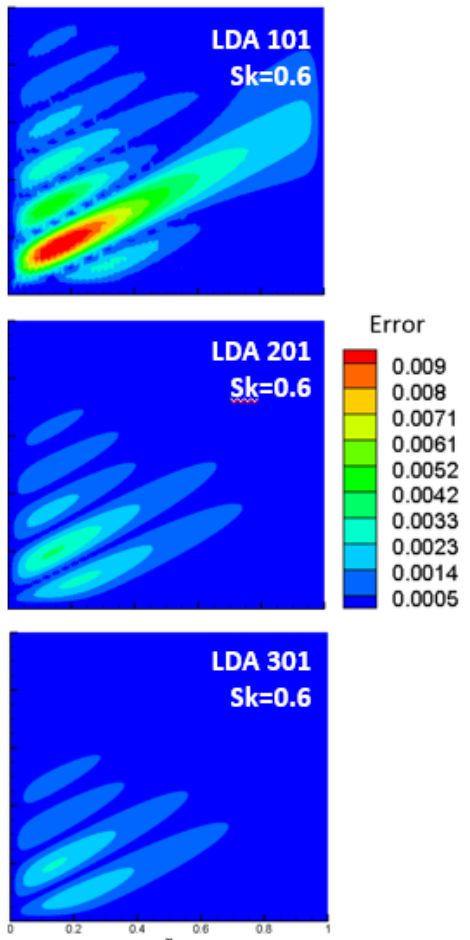
On the other hand, the Flux-Difference cases produce the opposite pattern where the error increases as the skewness increasing which demonstrates by Figure 6 . This pattern also can be seen on the finer grid. But by comparing both cases at skewness 0.0 , the Flux-Difference case has less error distribution as the error contours distribution are smaller and narrower. This is due to the FluxDifferences has preserved a second order accurate whereby the classic LDA only first order accurate. These patterns were proved on Figure 7 where the graph shows the order of accuracy for both cases with different skewness.
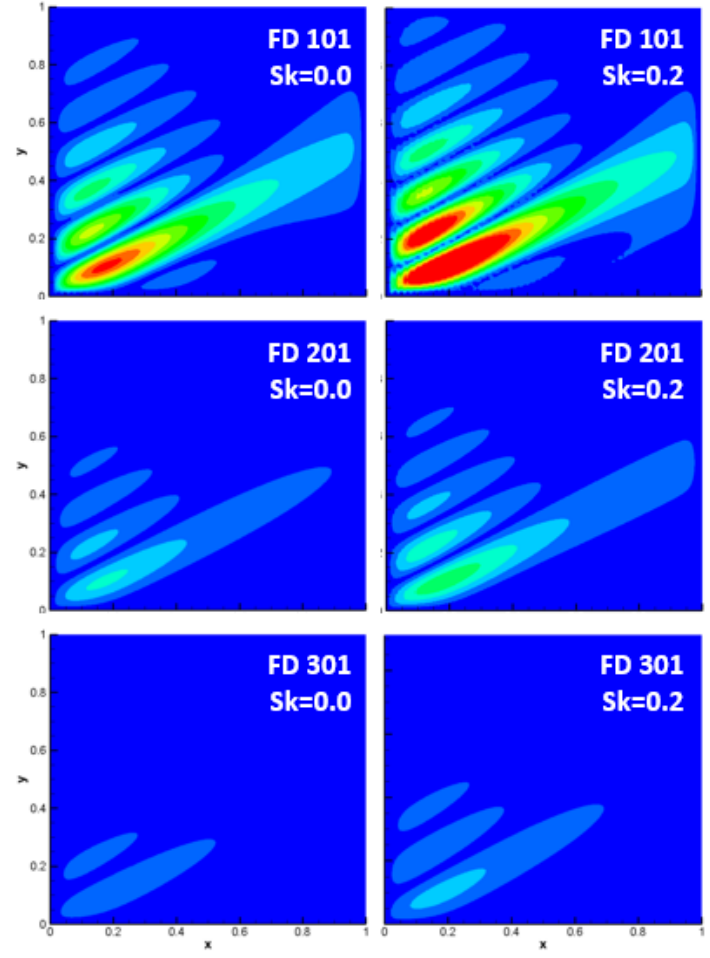

Fig. 6. Error contour for Flux-Difference cases
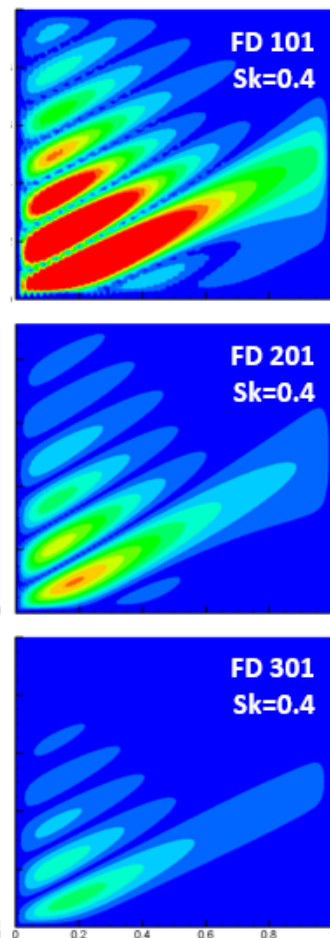
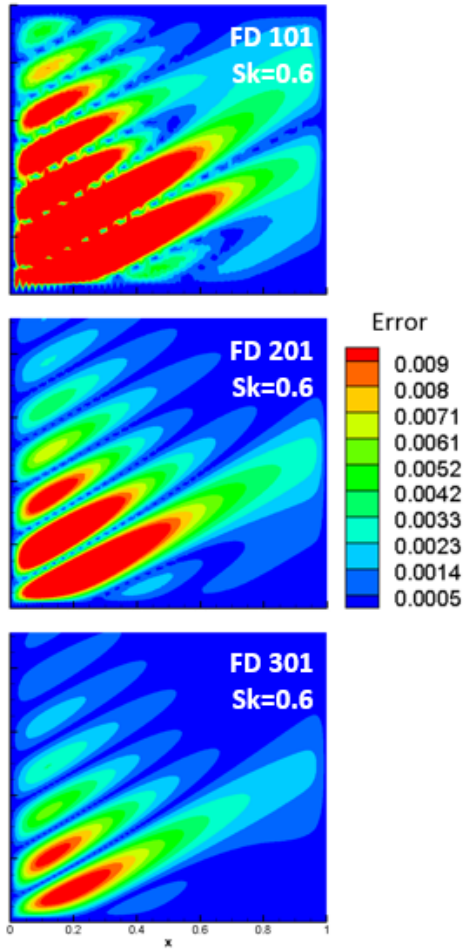

0.0005

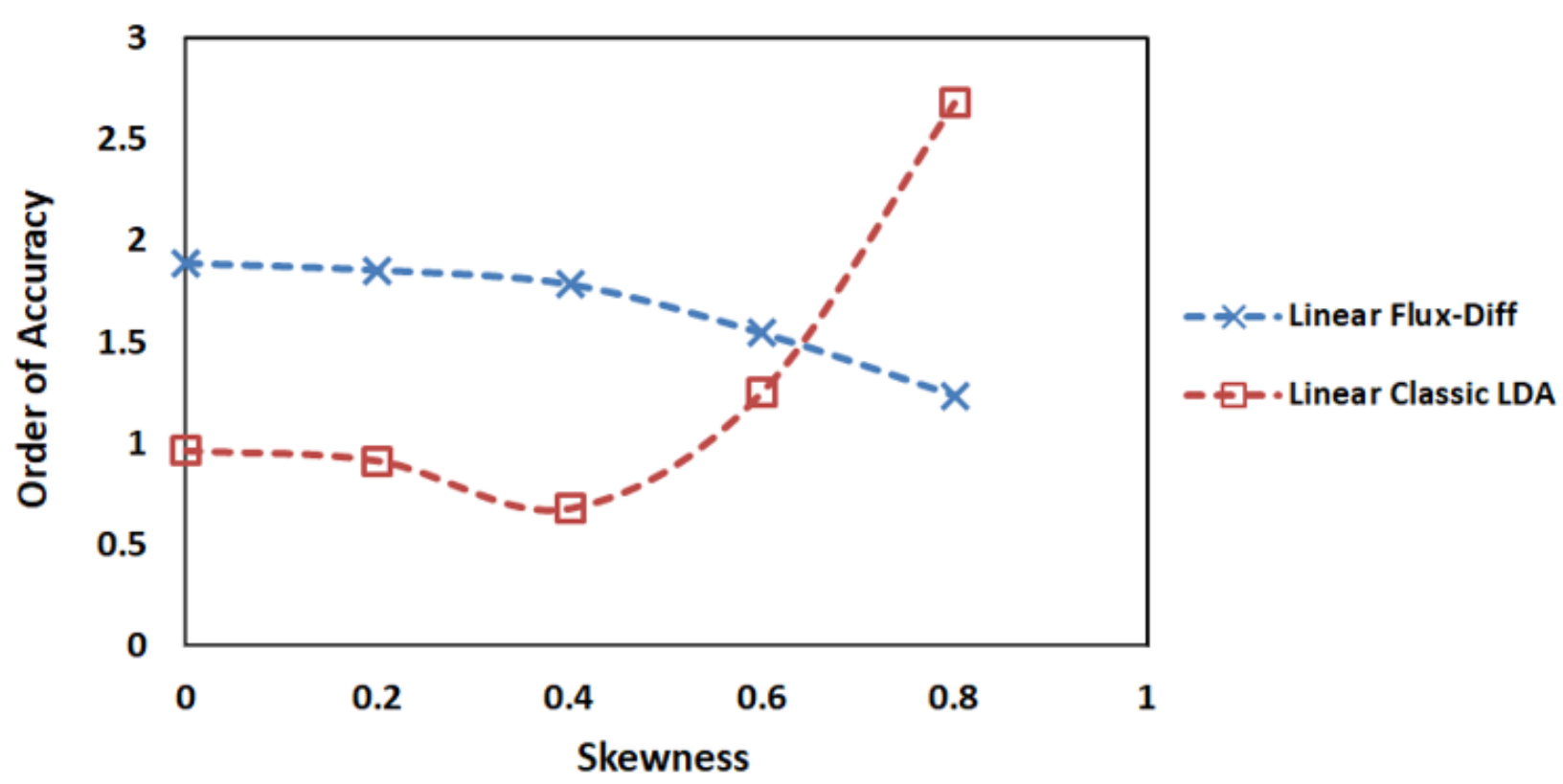

Fig. 7. The graph of Order of Accuracy vs Skewness 


\section{Conclusions}

The present study involves a numerical investigation on the effect of grid skewness on non-unified compact residual-distribution methods for scalar advection-diffusion problems. 2 test cases, the classic RD (LDA) and Flux-Difference approach have been tested using equilateral grid with several grid skewness values. From the results, it can be concluded that

i. The Flux-Difference approach shows a better accuracy compared to the classic RD approach where the results demonstrate to be second order accurate without using additional equations for the range of 0.0 to 0.4 skewness.

ii. This has been proven by mathematical formulation in Ref. [5], at least for skewness 0.0. However, the Flux-Difference approach suffers a marked accuracy drop beyond skewness 0.6, which is beyond the usual grid skewness limit of practical CFD.

iii. On the classic RD case, the effect of grid skewness have non-uniform pattern where the order of accuracy is dropping from grid skewness 0.0 till 0.4 . however, the accuracy has increases as the grid highly skewed (0.6 and 0.8 )

iv.Meanwhile, the Flux-Difference case has a more uniform pattern as the order of accuracy decreases corresponding to the high skewed grids.

\section{Acknowledgement}

This research was funded by a grant from Ministry of Higher Education of Malaysia (FRGS Grant VOT 1545).

\section{References}

[1] Norhaliza Abu Bakar, Rozaini Roslan and Arash Karimipour. "Magnetic Field Effect on Mixed Convection Heat Transfer in a Lid-Driven Rectangular Cavity." CFD Letters 12, no. 1 (2020): 13-21.

[2] Zakaria, Mohamad Shukri, Farzad Ismail, Masaaki Tamagawa, Ahmad Fazli Abdul Azi, Surjatin Wiriadidjaya, Adi Azrif Basri, and Kamarul Arifin Ahmad. "Computational Fluid Dynamics Study of Blood Flow in Aorta using OpenFOAM." Journal of Advanced Research in Fluid Mechanics and Thermal Sciences 43, no. 1 (2018): 81-89.

[3] Guzik, S. M. J., and C. P. T. Groth. "Comparison of solution accuracy of multidimensional residual distribution and Godunov-type finite-volume methods." International Journal of Computational Fluid Dynamics 22, no. 1-2 (2008): 61-83. https://doi.org/10.1080/10618560701748176

[4] Ismail, Farzad, Pablo M. Carrica, Tao Xing, and Frederick Stern. "Evaluation of linear and nonlinear convection schemes on multidimensional non-orthogonal grids with applications to KVLCC2 tanker." International journal for numerical methods in fluids 64, no. 8 (2010): 850-886.

https://doi.org/10.1002/fld.2174

[5] Katz, Aaron, and Venkateswaran Sankaran. "High aspect ratio grid effects on the accuracy of Navier-Stokes solutions on unstructured meshes." Computers \& fluids 65 (2012): 66-79. https://doi.org/10.1016/i.compfluid.2012.02.012

[6] Roe, Philip L. "Characteristic-based schemes for the Euler equations." Annual review of fluid mechanics 18, no. 1 (1986): 337-365.

https://doi.org/10.1146/annurev.fl.18.010186.002005

[7] Chizari, Hossain, and Farzad Ismail. "Accuracy variations in residual distribution and finite volume methods on triangular grids." Bulletin of the Malaysian Mathematical Sciences Society 40, no. 3 (2017): 1231-1264. https://doi.org/10.1007/s40840-015-0292-0

[8] Chizari, Hossain, and Farzad Ismail. "A grid-insensitive Ida method on triangular grids solving the system of euler equations." Journal of Scientific Computing 71, no. 2 (2017): 839-874.

https://doi.org/10.1007/s10915-016-0323-5

[9] Abgrall, Remi, and J. Trefilik. "An example of high order residual distribution scheme using non-lagrange elements." Journal of Scientific Computing 45, no. 1-3 (2010): 3-25.

https://doi.org/10.1007/s10915-010-9405-y 
[10] Mazaheri, Alireza, and Hiroaki Nishikawa. "Improved second-order hyperbolic residual-distribution scheme and its extension to third-order on arbitrary triangular grids." Journal of Computational Physics 300 (2015): 455-491. https://doi.org/10.1016/j.jcp.2015.07.054

[11] Deconinck, H., Philip L. Roe, and R. Struijs. "A multidimensional generalization of Roe's flux difference splitter for the Euler equations." Computers \& fluids 22, no. 2-3 (1993): 215-222. https://doi.org/10.1016/0045-7930(93)90053-C

[12] Abgrall, Remi. "A review of residual distribution schemes for hyperbolic and parabolic problems: the July 2010 state of the art." Communications in Computational Physics 11, no. 4 (2012): 1043-1080. https://doi.org/10.4208/cicp.270710.130711s

[13] Ismail, Farzad, and Hossain Chizari. "Developments of entropy-stable residual distribution methods for conservation laws I: scalar problems." Journal of Computational Physics 330 (2017): 1093-1115. https://doi.org/10.1016/i.jcp.2016.10.065

[14] Nishikawa, Hiroaki. "A first-order system approach for diffusion equation. I: Second-order residual-distribution schemes." Journal of Computational Physics 227, no. 1 (2007): 315-352. https://doi.org/10.1016/i.jcp.2007.07.029

[15] Nishikawa, Hiroaki, and Philip Roe. "On high-order fluctuation-splitting schemes for Navier-Stokes equations." In Computational fluid dynamics 2004, pp. 799-804. Springer, Berlin, Heidelberg, 2006. https://doi.org/10.1007/3-540-31801-1 116

[16] Nishikawa, Hiroaki. "A first-order system approach for diffusion equation. II: Unification of advection and diffusion." Journal of Computational Physics 229, no. 11 (2010): 3989-4016. https://doi.org/10.1016/j.jcp.2009.10.040

[17] Singh, Vishal, Hossain Chizari, and Farzad Ismail. "Non-unified compact residual-distribution methods for scalar advection-diffusion problems." Journal of Scientific Computing 76, no. 3 (2018): 1521-1546. https://doi.org/10.1007/s10915-018-0674-1 